\title{
Characteristics of childhood allergic diseases in outpatient and emergency departments in Shanghai, China, 2016- 2018: a multicenter, retrospective study
}

Yuanyuan $\mathrm{Qi}^{1}$, Peng Shi ${ }^{2}$, Renjie Chen ${ }^{3}$, Yufeng Zhou ${ }^{4,5}$, Lijuan Liu' ${ }^{1}$, Jianguo Hong ${ }^{6}$, Lanfang Cao ${ }^{7}$, Yanming Lu', Xiaoyan Dong', Jing Li ${ }^{10}$, Yu Shi ${ }^{10}$, Min Xia ${ }^{7}$, Bo Ding $^{8}$, Liling Qian', Libo Wang ${ }^{1}$, Wenhao Zhou ${ }^{11}$,

Yonghao Gui ${ }^{12}$ and Xiaobo Zhang ${ }^{*}$

\begin{abstract}
Background: The prevalence of allergic diseases (ADs), such as asthma and allergic rhinitis (AR), is increasing worldwide in both adults and children. Although ADs are common and frequently coexist in outpatient care, citylevel data regarding the characteristics of childhood AD remain limited in China. This study aimed to assess the profile and characteristics of ADs in the city of Shanghai.

Methods: A multicenter retrospective study was designed to collect routine administrative data from outpatient and emergency departments from 66 hospitals in Shanghai, China, from 2016 to 2018. Children with asthma, AR, allergic conjunctivitis (AC), and allergic skin diseases were investigated. Demographic characteristics, patients visit pattern, spectrum of diagnosis, and comorbidities were analyzed.
\end{abstract}

Results: A total of 2,376,150 outpatient and emergency visits for ADs were included in the period from 2016 to 2018. Allergic skin diseases accounted for 38.9\%, followed by asthma (34.8\%), AR (22.9\%), and AC (3.3\%), with a male predominance in all four diseases. Asthma and allergic skin diseases were most frequent in the 1 to $<4$ years of age group, while AR and AC were more common in the 4 to $<7$ years of age group. Asthma accounted for the greatest number of annual and emergency visits. The most frequent comorbidity of asthma was lower respiratory tract infection (LRTI) (49.3\%), followed by AR (20.5\%) and upper respiratory tract infection (14.1\%). The most common comorbidities of AR were otitis media (23.4\%), adenoid hypertrophy/obstructive sleep apnea (22.1\%), followed by LRTI (12.1\%), asthma (9.4\%) and chronic pharyngitis (8.9\%).

Conclusions: Asthma and allergic skin diseases were the most common ADs in outpatient and emergency departments in the study period. Respiratory tract infection was the most common comorbidity of asthma in children. More attention should be devoted to the treatment of comorbidities to improve childhood AD outcomes with a better understanding of the characteristics of ADs in outpatient care.

Keywords: Allergic diseases, Asthma, Allergic rhinitis, Comorbidity, Outpatient visit

\footnotetext{
* Correspondence: zhangxiaobo0307@163.com

'Department of Respiratory Medicine, Children's Hospital of Fudan University,

National Children's Medical Center, Shanghai 201102, China

Full list of author information is available at the end of the article
}

\section{$\triangle B M C$}

C C The Author(s). 2021 Open Access This article is licensed under a Creative Commons Attribution 4.0 International License, which permits use, sharing, adaptation, distribution and reproduction in any medium or format, as long as you give appropriate credit to the original author(s) and the source, provide a link to the Creative Commons licence, and indicate if changes were made. The images or other third party material in this article are included in the article's Creative Commons licence, unless indicated otherwise in a credit line to the material. If material is not included in the article's Creative Commons licence and your intended use is not permitted by statutory regulation or exceeds the permitted use, you will need to obtain permission directly from the copyright holder. To view a copy of this licence, visit http://creativecommons.org/licenses/by/4.0/ The Creative Commons Public Domain Dedication waiver (http://creativecommons.org/publicdomain/zero/1.0/) applies to the data made available in this article, unless otherwise stated in a credit line to the data. 


\section{Background}

Allergic diseases (ADs) represent one of the most common types of respiratory diseases globally, not only in adults, but also in children and adolescents [1]. Over the past decades, the prevalence of ADs has increased worldwide [2,3]. The complexity and severity of ADs continue to increase, especially in children, resulting in substantial healthcare expenditures and a high burden on patient' quality of life [4].

The prevalence of ADs varies greatly according to geographical region. Huang et al. [5] reported a prevalence of $10.2 \%$ for asthma, $54.1 \%$ for rhinitis, and $22.5 \%$ for eczema in Shanghai, China. A retrospective study conducted in a tertiary pediatric hospital in Shanghai reported that asthma and rhinitis accounted for $20 \%$ of respiratory outpatient visits [6]. ADs are often associated with various comorbidities, which have a significant impact on health outcomes [7-10]. Although ADs are common and frequently coexist in outpatient care, data regarding the characteristics of childhood AD have remained limited in China for a prolonged period. Understanding the profile and characteristics of ADs can provide insight into local health policies and guide clinical practice.

The primary objective of the present study was to describe the characteristics of ADs (asthma, allergic rhinitis [AR], allergic skin diseases, and allergic conjunctivitis $[\mathrm{AC}]$ ) in selected outpatient and emergency departments in Shanghai, China, and comorbidities of ADs.

\section{Methods}

\section{Study design}

A multicenter, retrospective study was designed to collect routine administrative data from two children hospitals and 64 general hospitals with pediatric clinics that provide the comprehensive services for children in Shanghai, China. Data regarding visits from children $\leq 18$ years of age, who were diagnosed with ADs, were collected from the hospital information system (HIS) of the outpatient department and emergency department (ED) between January 1, 2016, and December 31, 2018. Demographic information, such as age, sex, date of birth, medical records of date of visit, and diagnosis, were included. Patient age was calculated as the difference between birth date and visit date, and classified as $<1,1$ to $<4,4$ to $<7,7$ to $<12$, and 12 to $\leq 18$ years. The study protocol was approved by the Research Ethics Committee of the Children's Hospital affiliated to Fudan University (Shanghai, China; NO. 2018-215). All protocols were performed in accordance with the relevant guidelines and regulations.

\section{Definitions of conditions}

The most common allergic disorders among children were investigated, including asthma, AR, allergic skin diseases, and AC. Allergic skin diseases in the study included atopic dermatitis, allergic contact dermatitis, urticaria, angioedema, and drug eruption. The International Classification of Diseases, 10th Revision codes (ICD-10), and detailed codes for each disease are shown in supplementary Table 1.

Cough variant asthma (CVA) was diagnosed according to the following criteria: clinical history of a persistent cough ( $\geq 4$ weeks) and airway high reaction, but no wheezing or dyspnea, in contrast to classic asthma, antiasthma drugs effective against their coughs, excluding other diseases that caused chronic cough.

Severe asthma attack was diagnosed according to criteria from the "Guideline for the diagnosis and optimal management of asthma in children (2016)" in China, which is characterized by respiratory distress, use of accessory muscles, tachypnea, tachycardia, difficulty with speaking in full sentences, decreased level of consciousness, oxygen saturation $90 \%$, and peak expiratory flow $\leq 50 \%$ of predicted for children $\geq 6$ years of age, and was characterized by respiratory distress, decreased level of consciousness, oxygen saturation $\leq 92 \%$, difficulty speaking in full sentences, cyanosis, or quiet chest for children $<6$ years of age.

Status asthmaticus was defined as a prolonged and severe asthma attack that did not respond to standard or optimal treatment.

Atopic dermatitis is a chronic inflammatory skin disease characterized by eczematous lesions and is associated with elevated serum immunoglobulin (Ig) E levels, and tissue and blood eosinophilia.

Diagnostic tests, including peripheral eosinophils, serum total and specific IgE, and/or skin prick tests, were performed by the healthcare providers according to clinical conditions.

\section{Statistical analyses}

Python was used for data processing and SPSS version 22.0 (IBM Corporation, Armonk, NY, USA) for data analysis. For descriptive analyses, numbers and percentages (\%) were used for qualitative variables. The chisquared test was used to test differences in proportions between the groups. The average annual growth rate (AAGR) was calculated in terms of the geometric growth, expressed as $\left(\mathrm{X}_{n} / \mathrm{X}_{0}\right)^{(1 / n)}-1$ (where $\mathrm{X}_{0}$ is the value of the baseline and $\mathrm{X}_{n}$ is the value of the $n$th year).

The missing data imputation were not applied in this study for the administrative data when the missing rate is low (Supplementary Table 2).

Differences with a two-tailed $P$ value $\leq 0.05$ was considered to be statistically significant. Due to the large sample sizes in this study, effect sizes were more meaningful than $P$ values in the interpretation of the results. 


\section{Results}

\section{Overview of visits from patients with ADs}

There were a total of 2,376,150 outpatient and emergency visits for ADs in the 66 hospitals from 2016 to 2018 in Shanghai. The total number of visits increased from 657,217 in 2016 to 917,565 in 2018, corresponding to an AAGR of $13.2 \%$. Among ADs, allergic skin diseases accounted for $38.9 \%$, followed by asthma (34.8\%), AR (22.9\%), and AC (3.4\%) (Table 1).

\section{Demographic characteristics of patients with ADs}

The proportions of sex, payer type, and age groups were compared among the four disease groups (Table 2). Male predominance was found in all four diseases. A lower male-to-female ratio was observed in allergic skin diseases than in asthma, AR, and AC (1.2 for allergic skin diseases, 1.9 for asthma, 1.7 for $\mathrm{AR}$, and 1.8 for $\mathrm{AC}$, $P<0.001)$.

For payer type, the proportion of children with government insurance was lower in allergic skin diseases (53.2\%) than in the other diseases $(65.2 \%$ for asthma, $64.3 \%$ for $\mathrm{AR}$, and $68.2 \%$ for $\mathrm{AC}, P<0.001)$.

Regarding the age distribution of ADs, in patients with asthma, the 1 to < 4 years' age group (37.9\%) was the most common, followed by the 4 to $<7(32.4 \%), 7$ to $<$ $12(16.2 \%),<1(9.6 \%)$, and 12 to $\leq 18(3.9 \%)$ years' age group. The 1 to < 4 years' age group (33.4\%) was most common in allergic skin diseases, followed by $<1$ (26.2\%), 4 to $<7(18.4 \%)$, and 7 to $<12(14.8 \%)$ years' age groups. The 4 to $<7$ years' age group was most common in $\mathrm{AR}$ and $\mathrm{AC}$, followed by the 7 to $<12,1$ to $<4$, and $<1$ years' age groups.

\section{Visit patterns of patients with ADs}

The proportion of ED visits was highest for asthma (19.8\%), followed by allergic skin diseases (8.1\%), AR (4.1\%), and AC (2.1\%). Demographic characteristics between outpatient visits and ED visits were compared (Supplementary Table 3). The number of visits by different age groups, genders, and payer types in the ED visits were found to be of a similar order as the outpatient department visits. For asthma, the proportion of boys was higher in ED visits than in outpatient visits. In contrast, the proportion of girls was higher in ED visits than in outpatient visits for AR and allergic skin diseases. The
ED visit ratio for asthma was highest in the 7 to $<12$ years' age group, followed by the 12 to $\leq 18,1$ to $<4$ years' age group, the $<1$ years' age group had the lowest ED visit ratio. The ratio of ED visits increased with age in the AR. The proportion of those with medical insurance was higher in ED visits than in outpatient visits for asthma, AR, and allergic skin diseases.

Seasonal visits according to month are presented in Fig. 1. The number of visits for asthma was lowest in February, increasing slightly in the spring, reduced in the summer, increased rapidly in September, and then reached a peak in November and December. Visits for AR were high in November and December and low from January to March. Visits for allergic skin diseases were high in July and August and low in January and February. Visits for AC were stable throughout the whole year, with a slight increase from May to July.

\section{Spectrum and comorbidities of ADs}

Among the visits for asthma, the proportion of CVA was 9.5\%, with an increase from 2016 to 2018 (Table 3). Asthma complicating respiratory infection accounted for $3.7 \%$ of total asthma visits, and with a decrease observed from 2016 to 2018. A total of 2636 (0.3\%) visits were for severe asthma attacks, and 62 patients were diagnosed with status asthmaticus.

Furthermore, patient characteristics were compared between those with classic asthma and CVA. The proportion of ED visits was lower for CVA (9.7\%) than for classic asthma (21.3\%). The number of visits was highest in 4 to $<7$ years' age group (46.9\%) for CVA and in the 1 to < 4 years' group for classic asthma. The male-tofemale ratio was lower in patients with CVA than in those with classic asthma.

Atopic dermatitis (70.8\%) was the most common allergic skin disease, followed by acute urticaria (27\%). Other allergic skin diseases included chronic urticaria $(0.895 \%)$, contact dermatitis $(0.624 \%)$, and angioedema $(0.506 \%)$. The proportion of drug eruptions was found to be $0.038 \%$. For atopic dermatitis, < 1-year-old group (32.8\%) was the most common, followed by 1 to $<4(30.4 \%), 4$ to $<7$ (16.7\%), 7 to $<12(13.9 \%)$, and 12 to $\leq 18(6.2 \%)$ years' age groups.

The comorbidities of asthma and CVA were analyzed separately. Of the 747,597 visits for asthma in the 3 years, 37,693 (5.0\%) had comorbidities (Fig. 2).

Table 1 Overview of visits from patients with allergic diseases in Shanghai, China, 2016 to 2018

\begin{tabular}{|c|c|c|c|c|}
\hline Diseases & 2016 & 2017 & 2018 & Total \\
\hline Asthma, $n(\%)$ & 267,091 (40.6) & $260,887(32.6)$ & $298,939(32.6)$ & $826,917(34.8)$ \\
\hline $\mathrm{AR}, n(\%)$ & 120,196 (18.3) & $179,142(22.3)$ & $243,873(26.5)$ & $543,211(22.9)$ \\
\hline Allergic skin diseases, $n(\%)$ & $249,596(38.0)$ & $333,284(41.6)$ & $341,857(37.3)$ & $924,737(38.9)$ \\
\hline$A C, n(\%)$ & $20,334(3.1)$ & 28,055 (3.5) & $32,896(3.6)$ & 81,285 (3.4) \\
\hline Total & 657,217 & 801,368 & 917,565 & $2,376,150$ \\
\hline
\end{tabular}


Table 2 Comparison of demographic characteristics of patients with allergic diseases

\begin{tabular}{|c|c|c|c|c|c|}
\hline Characteristic & Asthma & AR & Allergic skin diseases & $A C$ & $P$ value \\
\hline Gender & & & & & $<0.001$ \\
\hline Male & $541,755(65.5)$ & $342,530(63.1)$ & $513,255(55.5)$ & $52,600(64.7)$ & \\
\hline Female & $284,987(34.5)$ & $198,982(36.6)$ & $411,428(44.5)$ & $28,679(35.3)$ & \\
\hline Age range, years & & & & & $<0.001$ \\
\hline$<1$ & $78,989(9.6)$ & $14,373(2.6)$ & $241,955(26.2)$ & $2717(3.3)$ & \\
\hline $1 \sim<4$ & $313,363(37.9)$ & $133,686(24.6)$ & $309,163(33.4)$ & $22,697(27.9)$ & \\
\hline $4 \sim<7$ & $268,172(32.4)$ & $207,631(38.2)$ & $170,555(18.4)$ & $30,045(37.0)$ & \\
\hline $7 \sim<12$ & $134,371(16.2)$ & $144,785(26.7)$ & $137,207(14.8)$ & $22,881(28.1)$ & \\
\hline $12 \sim \leq 18$ & $32,022(3.9)$ & $42,736(7.9)$ & $65,857(7.1)$ & $2945(3.6)$ & \\
\hline Payer type & & & & & $<0.001$ \\
\hline Medical insurance & $539,264(65.2)$ & $349,116(64.3)$ & $492,112(53.2)$ & $55,400(68.2)$ & \\
\hline Self-finance & $284,987(34.8)$ & $194,095(36.7)$ & $432,625(46.8)$ & $25,885(31.8)$ & \\
\hline
\end{tabular}

The most common comorbidities of asthma were lower respiratory tract infection (LRTI) (49.3\%), AR (20.5\%), and upper respiratory tract infection (URTI) (14.1\%). Other allergic comorbidities included food allergies or others (2.98\%), allergic skin diseases
(1.51\%), and $\mathrm{AC}(0.75 \%)$. Moreover, a small percentage of patients also had co-conditions such as epilepsy/seizure disorder (0.18\%) and tic disorder (TD)/ attention deficit hyperactivity disorder (ADHD) (0.14\%).

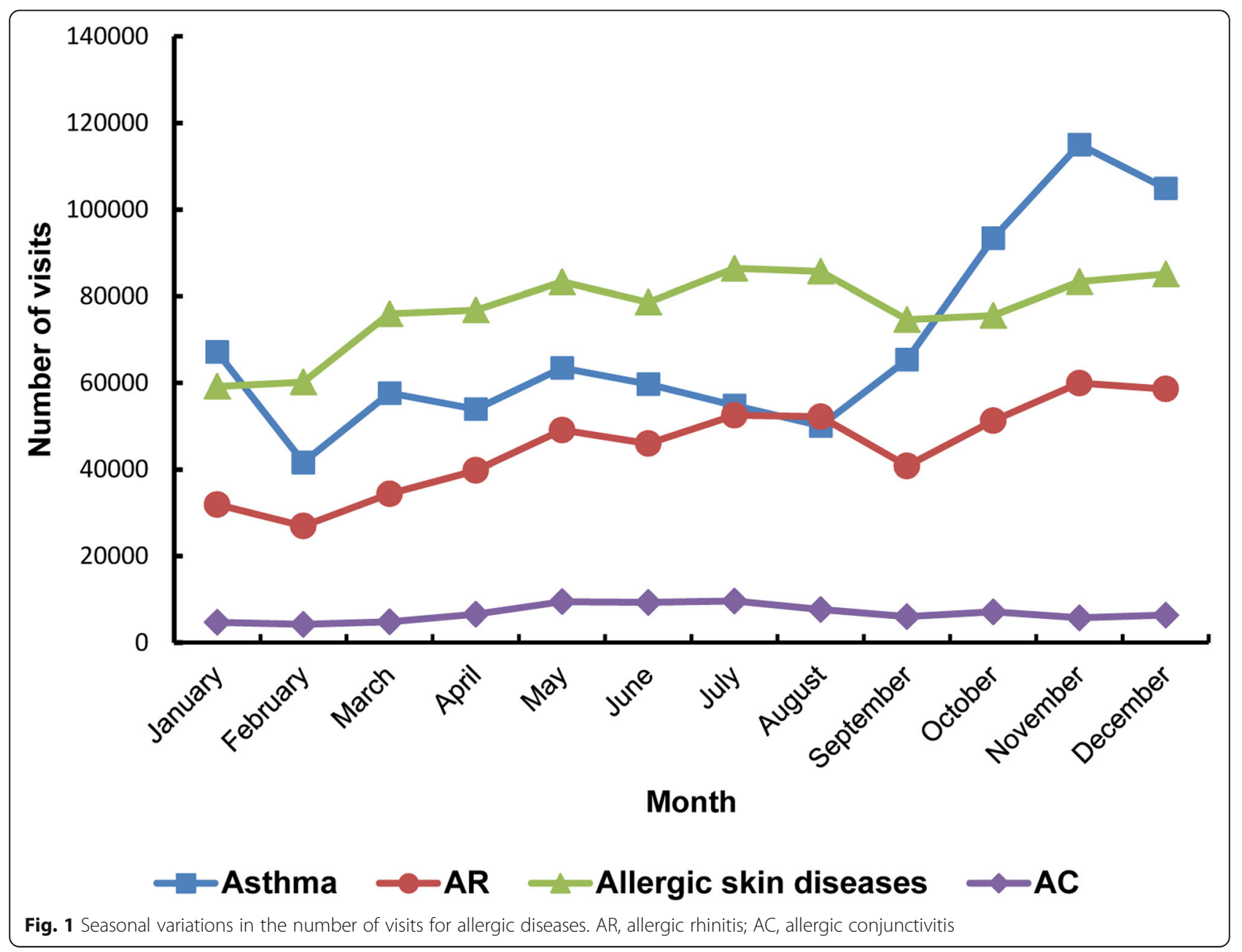


Table 3 Spectrum of diagnosis of asthma and allergic skin diseases

\begin{tabular}{|c|c|c|c|c|}
\hline Characteristic & 2016 & 2017 & 2018 & Total \\
\hline \multicolumn{5}{|l|}{ Asthma } \\
\hline Asthma alone & $237,480(88.9)$ & $233,497(89.5)$ & $243,975(81.6)$ & $714,952(86.5)$ \\
\hline Asthma complicated by respiratory infection & $14,361(5.4)$ & $7790(3.0)$ & $8275(2.8)$ & $30,729(3.7)$ \\
\hline Severe asthma attack & $570(0.2)$ & $781(0.3)$ & $1588(0.5)$ & $2636(0.3)$ \\
\hline Status asthmaticus & $24(0.009)$ & $26(0.010)$ & $12(0.004)$ & $62(0.007)$ \\
\hline Cough variant asthma & $14,620(5.5)$ & $18,736(7.2)$ & $45,047(15.7)$ & $78,403(9.5)$ \\
\hline \multicolumn{5}{|l|}{ Allergic skin disease } \\
\hline Atopic dermatitis & $176,885(70.9)$ & $242,312(72.7)$ & $235,712(69.0)$ & $654,909(70.8)$ \\
\hline Acute urticaria & $68,853(27.6)$ & $84,410(25.3)$ & $96,826(28.3)$ & $250,089(27.0)$ \\
\hline Chronic urticaria & $2406(0.96)$ & $2901(0.870)$ & $2974(0.870)$ & $8281(0.895)$ \\
\hline Contact dermatitis & $780(0.313)$ & $1861(0.558)$ & $3125(0.914)$ & $5766(0.624)$ \\
\hline Angioedema & $552(0.221)$ & $1465(0.440)$ & $2665(0.780)$ & $4682(0.506)$ \\
\hline Photosensitive dermatitis & $114(0.046)$ & $196(0.059)$ & $347(0.102)$ & $657(0.071)$ \\
\hline Drug Eruption & $6(0.002)$ & 139 (0.042) & $208(0.061)$ & $353(0.038)$ \\
\hline
\end{tabular}

Patients with CVA had a higher comorbidity rate than those with classic asthma. Of the all 78,403 visits for CVA, 22,281 (28.4\%) had coexisting conditions (Fig. 3). Compared with asthma, CVA had a higher proportion of coexisting LRTIs (63.1\%) and a lower proportion of URTIs (4.54\%). AR was the second most common comorbidity of CVA. Other allergic comorbidities included allergic pharyngolaryngitis $(0.96 \%)$, food allergy or others $(0.84 \%)$, obstructive sleep apnea (OSA) $(0.62 \%)$, allergic skin diseases $(0.46 \%)$, and $\mathrm{AC}(0.25 \%)$.

Of the 543,211 allergic rhinitis visits for 3 years, 78,266 (14.4\%) had any concomitant diseases (Fig. 4). The most common comorbidities were otitis media (23.4\%), adenoid hypertrophy/OSA (22.1\%), followed by LRTI

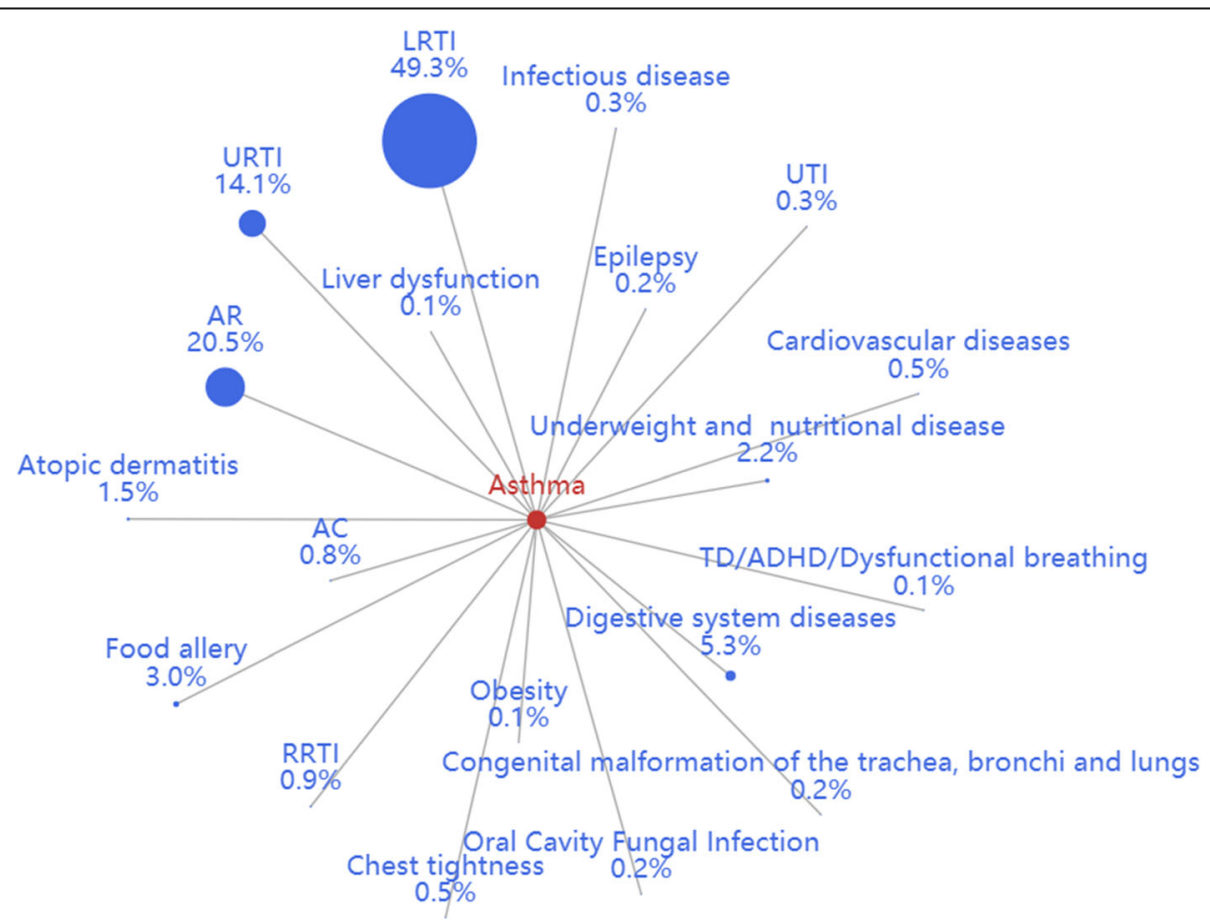

Fig. 2 The constituent ratio of comorbidities in asthma. Abbreviations: RRTI, recurrent respiratory tract infection; AC, allergic conjunctivitis; $A R$, allergic rhinitis; TD, tic disorder; ADHD, attention-deficit hyperactivity disorder; LRTI, lower respiratory tract infection; URTI, upper respiratory tract infection; UTI, urinary tract infection 


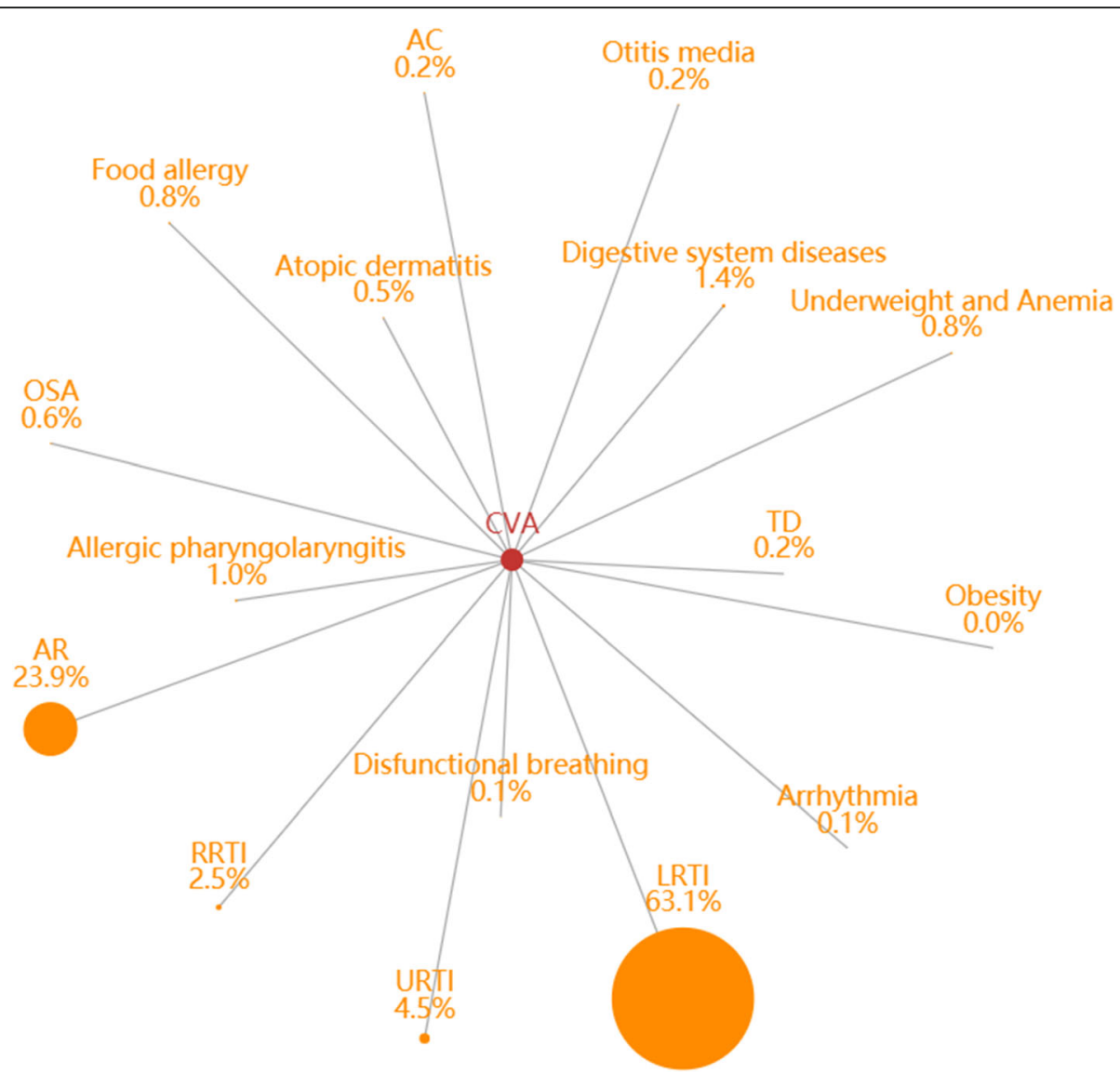

Fig. 3 The constituent ratio of comorbidities in cough variant asthma (CVA). Abbreviations: RRTI, recurrent respiratory tract infection; AC, allergic conjunctivitis; AR, allergic rhinitis; TD, tic disorder; LRTI, lower respiratory tract infection; URTI, upper respiratory tract infection; OSA, obstructive sleep apnea

(12.1\%), asthma (9.4\%), chronic pharyngitis (8.9\%), upper airway cough syndrome (UACS) (7.2\%) and rhinosinusitis $(6.5 \%)$. Other coexist allergic conditions included allergic skin diseases (0.56\%), allergic conjunctivitis (0.25\%).

\section{Discussion}

We reported a multicenter retrospective study to describe the characteristics of asthma, AR, allergic skin diseases and $\mathrm{AC}$ in pediatric outpatient and emergency settings in Shanghai, China.

The global prevalence of AD has increased dramatically in recent decades. However, the prevalence and spectrum of $\mathrm{AD}$ vary according to region and race. Alemayehu et al. and David et al. reported that the rank order of common ADs was asthma, AR, atopic dermatitis and $A C[11,12]$. A study from Korea reported that the most common $\mathrm{AD}$ was asthma, followed by atopic dermatitis, AR, and AC [13]. In the present study, allergic skin diseases were the most frequent, followed by asthma and AR. The different prevalences of ADs among areas may be attributed to differences in genetic background and environmental factors. We did not include the data on food allergy and drug hypersensitivity due to the lack of pediatric allergy clinics; nevertheless, further research is needed.

In our study, the proportion of AR among ADs increased significantly from 2016 to 2018 . There may be several reasons for this finding, the first of which is that outdoor and indoor environmental pollution may be associated with the increased prevalence of AR. Second, due to intensive training for pediatricians in asthma and $\mathrm{AR}$, more patients were diagnosed in a timely manner.

In our study, there was only a small proportion of patients with $\mathrm{AC}$, and was lower than that reported in other studies [11-13]. There may be several reasons for this difference. First, the majority of patients experienced few episodes of mild and intermittent symptoms. Second, only a few of the medical settings had ophthalmology outpatient departments for children. More attention should be devoted to the diagnosis and treatment of $\mathrm{AC}$ in the future.

It is well known that ADs have a natural history, and the incidence of specific ADs varies according to age. A 


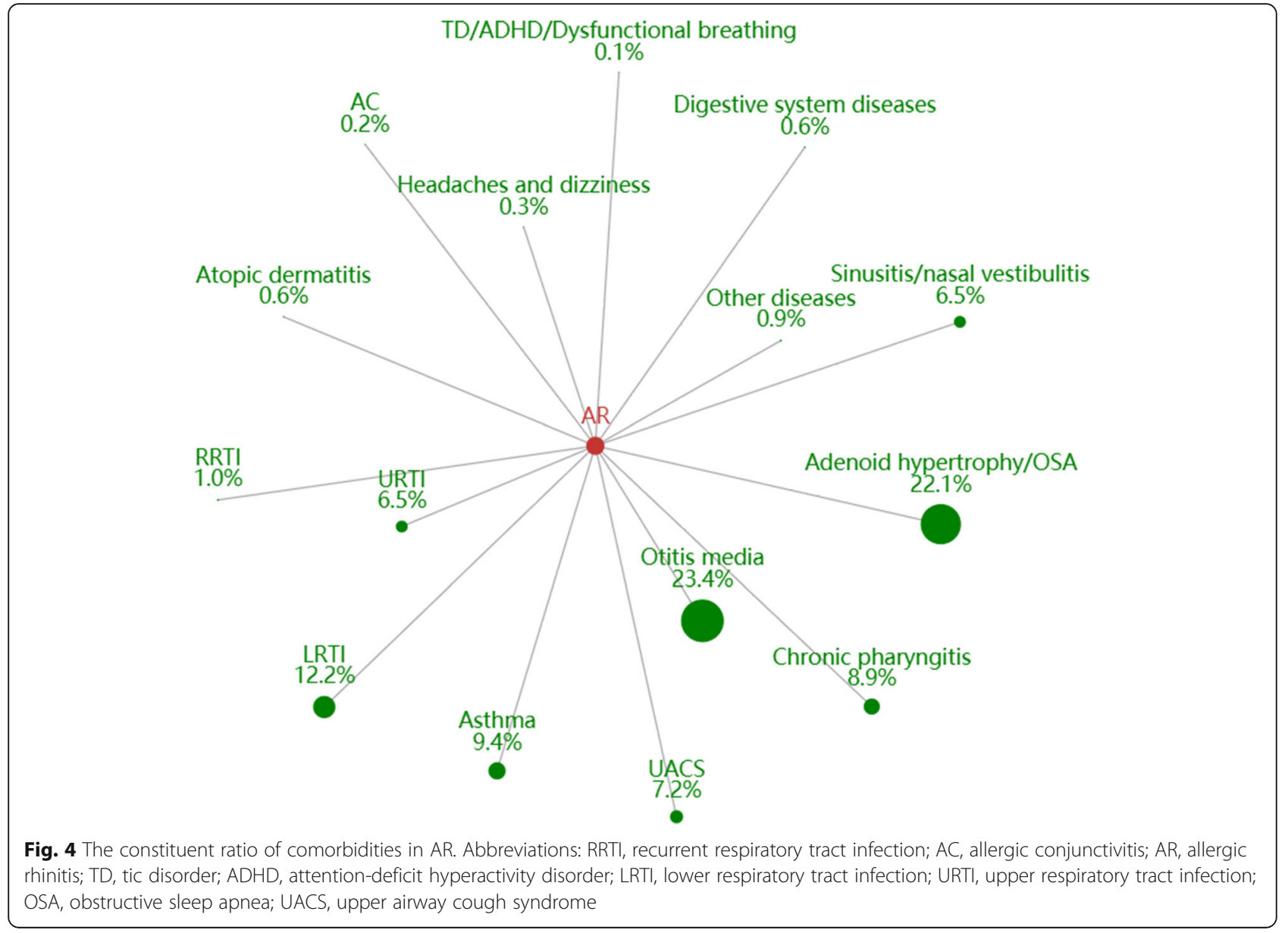

study involving a Swedish primary care asthma population reported that the number of asthma patients was significantly higher in the 7-14 years' group than in the $<7$ years' group [14]. An epidemiological study from the United States documented an increasing trend in the prevalence of asthma with age [12]. However, a recent meta-analysis reported the highest prevalence in children 5-9 years of age in 2010 in China [15]. In the present study, the number of visits for asthma was highest in the 1 to $<4$ and 4 to $<7$ years' group. A possible explanation for this inconsistency is the high incidence of respiratory infection in the 1 to < 4 years' group. The prevalence of atopic dermatitis is believed to decrease with increasing age [16]. In our study, atopic dermatitis was observed most commonly in the $<1$ and 1 to $<4$ years' groups, which was similar to previous reports $[17,18]$.

Our results suggest a higher prevalence of ADs in boys compared to girls. This is consistent with previous studies that reported a male predominance [19]. The overall percentage of medical insurance in allergic skin diseases group is lower than other allergic disease groups. This may be partly due to the delayed medical insurance procedures in infants by their parents. However, other factors contributing to this discrepancy, e.g. economic status, environmental factors, needs further investigation.

Seasonal variations in $\mathrm{AD}$ have been of great interest in clinical practice. However, there are few studies with large-scale epidemiological data. In the present study, clear seasonality was found in the four ADs, with evident differences among them.

Regarding asthma, many factors may influence the risk for visit for asthma [20]. It has been reported that asthma peaked in winter and spring and was negatively correlated with both temperature and humidity [21]. In this study, the increased visits for asthma in autumn and winter could be mainly caused by increased viral infection or cold temperatures. However, there was a sharp decrease in the number of visits in February, which may be due to the winter vacation when cross infections decreased.

Two types of seasonal patterns ("summer" type and "winter" type) of atopic dermatitis have been reported [22]. Noh et al. reported a negative association between atopic dermatitis symptoms and temperature [23]. Fleischer et al. found that increased temperatures predicted increased atopic dermatitis office visits [24]. The 
relative higher temperature and humidity in summer may contribute to the higher visits of allergic skin diseases in the present study.

Similar to a previous study, more visits for AR were observed in colder months in the present study. However, there were also high numbers of visits for AR from May to August, which may be associated with a corresponding increase in aeroallergens, such as pollen [25]. The outpatient visits for AC increased from May to July, which was consistent with a previously literature [26]. This may be explained by the increase of temperature and pollen.

Studies have also found that air pollution have adverse impact on ADs [27-29]. Further research is needed to ascertain the specific factors that explain the different seasonal variations of allergic diseases. Moreover, the visit trend may be associated with the total visit characteristics of outpatient and emergency departments, and further research should be conducted to evaluate its influence.

In the present study, we found that boys had a higher ED visit ratio than girls with asthma, which is consistent with other reports [30]. In this study, the ED ratios were the lowest in the $<1$ year group and increased with increasing age. There are conflicting reports in the literature regarding the influence of age on ED visits for asthma. Wasilewski et al. [31] reported that younger children exhibited a higher risk of ED visits. However, Tolomeo et al. [32] found that the risk of EV visits increase with age, which is consistent with our findings. This can be partly explained by different triggers of asthma exacerbations. Another possible explanation is that school-aged children are more likely to choose ED visit after school to avoid missing the school lessons.

CVA is easily overlooked and clinically misdiagnosed. In this study, we compared the clinical features of classic asthma and CVA. CVA had a lower ED visit and a lower male ratio. This is consistent with previous reports that females were more frequently affected by CVA than classic asthma, although mechanisms of sex differences remain unclear. Studies have documented that CVA has a shorter duration of disease and higher forced expiratory volume in $1 \mathrm{~s}$. Gao et al. [33] reported that patients with CVA experienced a shorter disease duration and higher lung function than patients with classic asthma. This may be the reason why CVA emergency visits were lower than those classic asthma. Patients with CVA had a higher proportion of ALRI than those with classic asthma. Mycoplasma pneumoniae infection has been reported to be related to the development of CVA. The pathogen in LRTI may be involved in CVA; however, the exact mechanism requires further research. We did not analyze the hospitalization rate and duration of hospitalization, and further research is needed to clarify these differences.
The findings that asthma and AR are associated with comorbid conditions have been previously demonstrated [34], and that comorbidities vary according to age group. LRTI was the most prevalent comorbidity in asthma in our study, whereas URTI was the most common comorbidity in all age group studies [14]. Gastroesophageal reflux disorder (GERD) is known to coexist with asthma [35]. However, the percentage of GERD in comorbidities was very low in the present study, suggesting that this comorbidity may be underestimated and undertreated in outpatient care for asthma. For AR, the most frequent comorbidities were otitis media and adenoid hypertrophy, while only a few percent of patients exhibited coexistent conjunctivitis and atopic dermatitis. It is noteworthy that $\mathrm{AC}$ was less common than AR in the present study than reported previously [36], partly because parents may have overlooked AC symptoms in their children. The association between neurobehavioral comorbidities and asthma or AR has been recently reported [37-40]. We also observed an association between TD/ADHD and ADs. The results indicated that more attention should be devoted to these co-conditions and should prompt further studies investigation the underlying mechanisms.

There were limitations to this study. The prevalence of ADs was not investigated in this study due to the lack of data for private medical institution visits and data of the catchment of hospitals. We did not include data regarding asthma and AR-related medication prescriptions, allergen immunotherapy, and the level of asthma control in asthma patients was not included in the present study. Therefore, further investigations should be conducted.

\section{Conclusion}

In summary, allergic skin diseases and asthma were the two major diseases in outpatient and emergency visits for ADs in children in Shanghai. Respiratory tract infection was the most common comorbidity of asthma. More attention should be devoted to early diagnosis and comprehensive treatment of ADs in children.

\begin{abstract}
Abbreviations
ADs: Allergic diseases; AR: Allergic rhinitis; AC: Allergic conjunctivitis; LRTI: Lower respiratory tract infection; OSA: Obstructive sleep apnea; HIS: Hospital information system; ED: Emergency department; ICD-10: Codes of International Classification of Disease, Revision 10; CVA: Cough variant asthma; TD: Tic disorder; ADHD: Attention deficit hyperactivity disorder; URTI: Upper respiratory tract infection; GERD: Gastroesophageal reflux disorder; RRTI: Recurrent respiratory tract infection; ADHD: Attention-deficit hyperactivity disorder; UACS: Upper airway cough syndrome; UTT: Urinary tract infection
\end{abstract}

\section{Supplementary Information}

The online version contains supplementary material available at https://doi. org/10.1186/s12887-021-02880-0.

Additional file 1: Supplementary Table 1. The detailed ICD codes for diseases. 
Additional file 2: Supplementary Table 2. Missing rate of gender and distribution comparison of age and payer type between missing group and non-missing group.

Additional file 3: Supplementary Table 3. Comparison of demographic characteristics between outpatient and ED visits.

\section{Acknowledgements}

Not applicable.

\section{Authors' contributions}

Xiaobo Zhang conceptualized and designed the study, supervised all analyses, critically reviewed and revised the manuscript; Yuanyuan Qi conducted the data analyses, drafted and revised the manuscript; Peng Shi designed the study, coordinated and supervised data collection, and revised the manuscript; Lijuan Liu designed the study, coordinated and supervised data collection; Jianguo Hong, Lanfang Cao, Yanming Lu, Xiaoyan Dong, Min Xia and Bo Ding collected data and supervised data collection; Jing Li and Yu Shi assisted in data clearing and carried out the initial analyses; Liling Qian and Libo Wang critically reviewed the manuscript for study design and statistical analysis; Renjie Chen, Yufeng Zhou and Wenhao Zhou assisted in interpretation of the results and revised the manuscript; Yonghao Gui critically reviewed the manuscript for important intellectual content. The author(s) read and approved the final manuscript.

\section{Funding}

This study was funded by Shanghai Science and Technology Committee (grand number: 18411951700), Intelligent Medical Research Project of Shanghai Health and Family Planning Commission (grand number: 2018ZHYL0225) and Specific Disease Cohort Project of Children's Hospital of Fudan University (grand number: 2020ZBDL10)

\section{Availability of data and materials}

The data collected and analyzed during the current study are available from the corresponding author on reasonable request.

\section{Declarations}

\section{Ethics approval and consent to participate}

The study was approved by the Ethics approval was obtained from the Research Ethic Committee of Children's Hospital affiliated to Fudan University (NO. 2018-215). And due to the nature of retrospective study, a waiver of consent was obtained from the Research Ethic Committee of Children's Hospital affiliated to Fudan University. All methods were performed in accordance with the relevant guidelines and regulations.

\section{Consent for publication}

Not applicable.

\section{Competing interests}

The authors declare that they have no competing interests.

\section{Author details}

'Department of Respiratory Medicine, Children's Hospital of Fudan University, National Children's Medical Center, Shanghai 201102, China. ${ }^{2}$ Department of Data Management and Statistics, Children's Hospital of Fudan University, Shanghai, China. ${ }^{3}$ School of Public Health, Key Lab of Public Health Safety of the Ministry of Education and NHC Key Laboratory of Health Technology Assessment, Fudan University, Shanghai 200032, China. ${ }^{4}$ Institute of Pediatrics, Children's Hospital of Fudan University, Shanghai 201102, China. ${ }^{5}$ Institutes of Biomedical Sciences, Fudan University, Shanghai 200032, China. ${ }^{6}$ Department of Pediatrics, Shanghai General Hospital, Shanghai Jiaotong University, Shanghai 200080, China. ${ }^{7}$ Department of Pediatrics, Renji Hospital, Shanghai Jiao Tong University, Shanghai 200127, China. ${ }^{8}$ Department of Pediatrics, South Campus, Renji Hospital, Shanghai Jiao Tong University, Shanghai 201112, China. 'Department of Respiratory Medicine, Children's Hospital of Shanghai Jiaotong University, Shanghai 200040, China. ${ }^{10}$ Big Data Product Department, Wonders Information Co. Ltd., Shanghai, China.

${ }^{11}$ Department of Neonatology, Children's Hospital of Fudan University, National Children's Medical Center, Shanghai 201102, China. ${ }^{12}$ Cardiovascular
Center, Children's Hospital of Fudan University, National Children's Medical Center, Shanghai 201102, China.

Received: 28 January 2021 Accepted: 31 August 2021

Published online: 17 September 2021

\section{References}

1. Bloom B, Jones LI, Freeman G. Summary health statistics for U.S. children: National Health Interview Survey, 2012. Vital Health Stat 10. 2013;258(258):181.

2. Pawankar R. Allergic diseases and asthma: a global public health concern and a call to action. World Allergy Organ J. 2014;7(1):12.

3. Maziak W, Behrens T, Brasky TM, Duhme H, Rzehak P, Weiland SK, et al. Are asthma and allergies in children and adolescents increasing? Results from ISAAC phase I and phase III surveys in Münster, Germany. Allergy. 2003; 58(7):572-9.

4. Asher J, Pearce N. Global burden of asthma among children. Int J Tuberc Lung Dis. 2014;18:1269-78.

5. Huang C, Liu W, Hu Y, Zou Z, Zhao Z, Shen L, et al. Updated prevalences of asthma, allergy, and airway symptoms, and a systematic review of trends over time for childhood asthma in Shanghai, China. PLoS One. 2015;10(4): e0121577.

6. Shi P, Zhang X, Liu L, Tang L, Li J, Wang L, et al. Age- and gender-specific trends in respiratory outpatient visits and diagnoses at a tertiary pediatric hospital in China: a 10-year retrospective study. BMC Pediatr. 2020;20(1):115.

7. Blackman JA, Gurka MJ. Developmental and behavioral comorbidities of asthma in children. J Dev Behav Pediatr. 2007;28:92-9.

8. Macri F, Rossi FP, Lambiase C, di Castelbianco FB, Frassanito A. Psychological factors in childhood asthma. Pediatr Pulmonol. 2008;43:366-70.

9. Westman M, Stjärne P, Asarnoj A, Kull I, van Hage M, Wickman M, et al. Natural course and comorbidities of allergic and nonallergic rhinitis in children. J Allergy Clin Immunol. 2012;129:403-8.

10. Ibáñez MD, Valero AL, Montoro J, Jauregui I, Ferrer M, Dávila I, et al. Analysis of comorbidities and therapeutic approach for allergic rhinitis in a pediatric population in Spain. Pediatr Allergy Immunol. 2013;24(7):678-84.

11. Gezmu AM, Kung SJ, Shifa JZ, Nakstad B, Brooks M, Joel D, et al. Pediatric spectrum of allergic diseases and asthma in a tertiary level Hospital in Botswana: an exploratory retrospective cross-sectional study. J Asthma Allergy. 2020;13:213-23.

12. Hill DA, Grundmeier RW, Ram G, Spergel JM. The epidemiologic characteristics of healthcare provider-diagnosed eczema, asthma, allergic rhinitis, and food allergy in children: a retrospective cohort study. BMC Pediatr. 2016;16:133.

13. Kang SY, Song WJ, Cho SH, Chang YS. Time trends of the prevalence of allergic diseases in Korea: a systematic literature review. Asia Pac Allergy. 2018;8(1):e8.

14. Lisspers K, Janson C, Larsson K, Johansson G, Telg G, Thuresson M, et al. Comorbidity, disease burden and mortality across age groups in a Swedish primary care asthma population: an epidemiological register study (PACEHR). Respir Med. 2018;136:15-20.

15. Li X, Song P, Zhu Y, Lei H, Chan KY, Campbell H, et al. The disease burden of childhood asthma in China: a systematic review and meta-analysis. J Glob Health. 2020;10(1):010801.

16. Sugiura $H$, Umemoto $N$, Deguchi $H$, Murata $Y$, Tanaka $K$, Sawai $T$, et al. Prevalence of childhood and adolescent atopic dermatitis in a Japanese population: comparison with the disease frequency examined 20 years ago. Acta Derm Venereol. 1998;78:293-4.

17. Lee JY, Yang HK, Kim M, Kim J, Ahn K. Is the prevalence of atopic dermatitis in Korean children decreasing?: National Database 2009-2014. Asian Pac J Allergy Immunol. 2017;35(3):144-9.

18. Guo Y, Li P, Tang J, Han X, Zou X, Xu G, et al. Prevalence of atopic dermatitis in Chinese children aged 1-7ys. Sci Rep. 2016;6:29751.

19. Postma DS. Gender differences in asthma development and progression. Gend Med. 2007:4(Suppl B):S133-46.

20. Di Cicco ME, Ferrante G, Amato D, Capizzi A, De Pieri C, Ferraro VA, et al. Climate change and childhood respiratory health: a call to action for paediatricians. Int J Environ Res Public Health. 2020;17(15):5344.

21. Lee YC, Ju HJ, Kwon JW, Bae JM. Seasonality of allergic diseases: real-world evidence from a nationwide population-based study. Immun Inflamm Dis. 2020;8(3):360-2. 
22. Krämer U, Weidinger S, Darsow U, Möhrenschlager M, Ring J, Behrendt H. Seasonality in symptom severity influenced by temperature or grass pollen: results of a panel study in children with eczema. J Invest Dermatol. 2005; 124(3):514-23.

23. Noh SR, Kim JS, Kim EH, Jeon BH, Kim JH, Kim YM, et al. Spectrum of susceptibility to air quality and weather in individual children with atopic dermatitis. Pediatr Allergy Immunol. 2019;30(2):179-87.

24. Fleischer $A B \mathrm{Jr}$. Atopic dermatitis: the relationship to temperature and seasonality in the United States. Int J Dermatol. 2019;58(4):465-71.

25. Chen BY, Chan CC, Han YY, Wu HP, Guo YL. The risk factors and quality of life in children with allergic rhinitis in relation to seasonal attack patterns. Paediatr Perinat Epidemiol. 2012;26(2):146-55.

26. Hong J, Zhong $\mathrm{T}$, Li H, Xu J, Ye X, Mu Z, et al. Ambient air pollution, weather changes, and outpatient visits for allergic conjunctivitis: a retrospective registry study. Sci Rep. 2016;6:23858.

27. Eguiluz-Gracia I, Mathioudakis AG, Bartel S, Vijverberg SJH, Fuertes E, Comberiati $\mathrm{P}$, et al. The need for clean air: the way air pollution and climate change affect allergic rhinitis and asthma. Allergy. 2020;75(9):2170-84.

28. Hu Y, Xu Z, Jiang F, Li S, Liu S, Wu M, et al. Relative impact of meteorological factors and air pollutants on childhood allergic diseases in Shanghai, China. Sci Total Environ. 2020;706:135975.

29. Min KD, Yi SJ, Kim HC, Leem JH, Kwon HJ, Hong S, et al. Association between exposure to traffic-related air pollution and pediatric allergic diseases based on modeled air pollution concentrations and traffic measures in Seoul, Korea: a comparative analysis. Environ Health. 2020;19(1):6.

30. Zhang $\mathrm{Q}$, Lamichhane R, Diggs LA. Disparities in emergency department visits in American children with asthma: 2006-2010. J Asthma. 2017;54(7): 679-86.

31. Wasilewski Y, Clark N, Evans D, Levison M, Levin B, Mellins RB. Factors associated with emergency department visits by children with asthma: implications for health education. Am J Pub Health. 1996;86:1410-5.

32. Tolomeo C, Savrin C, Heinzer M, Bazzy-Asaad A. Predictors of asthma-related pediatric emergency department visits and hospitalizations. J Asthma. 2009; 46(8):829-34.

33. Gao J, Wu F, Wu S, Yang X. Inflammatory subtypes in classic asthma and cough variant asthma. J Inflamm Res. 2020;13:1167-73.

34. Cazzola M, Calzetta L, Bettoncelli G, Novelli L, Cricelli C, Rogliani P. Asthma and comorbid medical illness. Eur Respir J. 2011;38(1):42-9.

35. Harding SM, Guzzo MR, Richter JE. The prevalence of gastroesophageal reflux in asthma patients without reflux symptoms. Am J Respir Crit Care Med. 2000;162:34-9.

36. Leonardi A, Castegnaro A, Valerio AL, Lazzarini D. Epidemiology of allergic conjunctivitis: clinical appearance and treatment patterns in a populationbased study. Curr Opin Allergy Clin Immunol. 2015;15(5):482-8,

37. Arif AA. The association between symptomatic asthma and neurobehavioral comorbidities among children. J Asthma. 2010;47(7):792-6.

38. Miyazaki C, Koyama M, Ota E, Swa T, Mlunde LB, Amiya RM, et al. Allergic diseases in children with attention deficit hyperactivity disorder: a systematic review and meta-analysis. BMC Psychiatry. 2017;17(1):120.

39. Lin YT, Chen YC, Gau SS, Yeh TH, Fan HY, Hwang YY, et al. Associations between allergic diseases and attention deficit hyperactivity/oppositional defiant disorders in children. Pediatr Res. 2016;80(4):480-5.

40. Feng $B$, Jin $H$, Xiang H, Li B, Zheng X, Chen R, et al. Association of pediatric allergic rhinitis with the ratings of attention-deficit/hyperactivity disorder. Am J Rhinol Allergy. 2017;31(3):161-7.

\section{Publisher's Note}

Springer Nature remains neutral with regard to jurisdictional claims in published maps and institutional affiliations.

Ready to submit your research? Choose BMC and benefit from:

- fast, convenient online submission

- thorough peer review by experienced researchers in your field

- rapid publication on acceptance

- support for research data, including large and complex data types

- gold Open Access which fosters wider collaboration and increased citations

- maximum visibility for your research: over $100 \mathrm{M}$ website views per year

At BMC, research is always in progress.

Learn more biomedcentral.com/submissions 